\title{
Hybrid classical-quantum computing: Applications to statistical mechanics of financial markets
}

\author{
Lester Ingber ${ }^{1^{*}}$ \\ ${ }^{1}$ Physical Studies Institute LLC, 7325 NE Imbrie Dr Ste 518, Hillsboro, OR 97124, USA
}

\begin{abstract}
Hybrid Classical-Quantum computing is now offered by several commercial quantum computers. In this project, a model of financial options, Statistical Mechanics of Financial Markets (SMFM), uses this approach. However, only Classical (super-)computers are used to include the quantum features of these models. Since 1989, Adaptive Simulated Annealing (ASA), an optimization code using importance-sampling, has fit parameters in such models. Since 2015, PATHINT, a path-integral numerical agorithm, has been used to describe several systems in several disciplines. PATHINT has been generalized from 1 dimension to $\mathrm{N}$ dimensions, and from classical to quantum systems into qPATHINT. Published papers have described the use of qPATHINT to neocortical interactions and financial options. The classical space modeled by SMFM fits parameters in conditional short-time probability distributions of nonlinear nonequilibrium multivariate statistical mechanics, while the quantum space modeled by qPATHINT describes quantum money. This project demonstrates how some hybrid classical-quantum systems may be calculated using only classical (super-)computers.
\end{abstract}

\section{Introduction}

\subsection{Hybrid computing}

There are several companies now offering commercial-grade Hybrid Classical-Quantum computers that can be accessed via the Cloud, e.g., Rigetti, D-Wave, Microsoft, and IBM [1]; see

https://docs.ocean.dwavesys.com/projects/hybrid/en/latest/index.html

https://www.rigetti.com/what

https://azure.microsoft.com/en-us/solutions/hybrid-cloud-app/\#overview

https://www.ibm.com/it-infrastructure/z/capabilities/hybrid-cloud

These companies typically offer Hybrid computing, consisting of Classical computers to run optimization program on parameters in systems that are described by quantum variables using their Quantum computers [2], Some studies show Quantum computing is still not possible for many systems, even with classical optimizers [3]. Software for quantum states,

* Corresponding author: ingber@caa.caltech.edu 
e.g., Tensorflow, the popular end-to-end open-source tool for machine learning, also has been adapted for Hybrid classical-quantum computing:

https://quantumzeitgeist.com/tensorflow-for-quantum-hits-first-birthday/

https://www.tensorflow.org/quantum

This project essentially codes generated for two previous XSEDE grants, "Electroencephalographic field influence on calcium momentum waves" and "Quantum path-integral qPATHTREE and qPATHINT algorithms". These codes run on Classical computers, defining a Hybrid Classical-Quantum solely on a Classical computer.

\subsection{SMFM}

For over two-score years, there have been occasional papers proposing Quantum Money (QM) [4-12]. There are several reasons to consider QM, including counterfeit-proof currency, and combining of currency with blockchain technologies yielding improved efficiencies and scaling beyond today's blockchains.

There is not yet a clear proposal for just how QM would be implemented or exchanged with classical money. However, quantum computing is rapidly growing, which will be applied in many ways to financial markets. Financial markets will be expanded to include quantum variables; financial markets will determine how QM is to be valued and how it may be exchanged with current financial instruments.

This paper does not address these problems in defining QM. Instead, this papaer addresses how options on such QM can be calculated.

"If" QM is not much of an issue. When QM arrives, options on quantum markets will be required for purposes of hedging and speculation.

Quantum options on quantum markets requires technologies similar to those required by options on classical financial markets. For example, American options, that can be exercised before maturation, prominent in today's markets, and these require numerical algorithms. As with today's technologies, probability distributions of prices in real markets will not generally be simple Gaussian or log-normal distributions that yield closed form options solutions. Seasonal changes and taxation issues require fits to determine actual distributions

\subsection{SMNI}

Previous papers since 1981 have have calculated properties of a model of neocortex, Statistical Mechanics of Neocortical Interactions (SMNI), to fit/describe many experimental data, e.g., electroencephalographic (EEG) data using a model of quantum wave-packets composed of a specific class of $\mathrm{Ca}^{2+}$ ions that are (re-)generated at tripartite neuronastrocyte-neuron sites, thereby influencing synaptic interactions [13-15].

The codes PATHTREE/qPATHTREE and PATHINT/qPATHINT can include random shocks in the evolution of a short-time probability distribution over thousands of foldings [16-19].

More details on how hybrid quantum-classical computing is being applied to this system currently is in another companion paper [20].

\subsection{PATHINT}

The path-integral can define a one-dimensional code developed to numerically propagate short-time conditional probability distributions [11,12,21], and this was generalized to PATHINT to an N-dimentional code. This was applied in several disciplines [22-25], and to PATHTREE [26]. 


\section{5 qPATHINT}

The PATHTREE and PATHINT codes were generalized to quantum systems, yielding qPATHTREE and qPATHINT $[16,17,19]$.

\subsection{Organization of paper}

Section 2 describes Adaptive Simulated Annealing (ASA) in the context of this project.

Section 3 describes PATHINT/qPATHINT in the context of this project.

Section 4 describes SMFM in the context of this project.

Section 5 describes performance and scaling issues.

Section 6 is the Conclusion.

\section{ASA Algorithm}

For parameters

$$
\alpha_{k}^{i} \in\left[A_{i}, B_{i}\right]
$$

sampling with the random variable $x^{i}$

$$
\begin{aligned}
x^{i} & \in[-1,1] \\
\alpha_{k+1}^{i} & =\alpha_{k}^{i}+x^{i}\left(B_{i}-A_{i}\right)
\end{aligned}
$$

the default generating function is

$$
g_{T}(x)=\prod_{i=1}^{D} \frac{1}{2 \ln \left(1+1 / T_{i}\right)\left(\left|x^{i}\right|+T_{i}\right)} \equiv \prod_{i=1}^{D} g_{T}^{i}\left(x^{i}\right)
$$

in terms of "temperatures" for parameters [27]

$$
T_{i}=T_{i 0} \exp \left(-c_{i} k^{1 / D}\right)
$$

ASA defaults use the same type of annealing schedule for the acceptance function $h$ as used for the generating function $g$, but all default functions can be overridden with userdefined functions [28,29].

Recently, ASA has been applied to studies of COVID-19, fitting forms like $x S^{y}$, for variables $S$ and parameters $x$ and $y$, in the drifts and covariances of conditional probability distributions [30].

ASA has over 150 OPTIONS to provide robust tuning over many classes of nonlinear stochastic systems. These many OPTIONS help ensure that ASA is robust across many systems.

The "QUENCHing" OPTIONS are among the most important for controlling Adaptive Simulated Annealing. Fuzzy ASA algorithms in particular offer ways of controlling how these QUENCHing OPTIONS may be applied across many classes of problems.

In the context of this project, ASA has an ASA_SAVE_BACKUP OPTIONS which periodically saves all information (including generated random numbers) sufficient to restart if it is interrupted, e.g., using the ASA_EXIT_ANYTIME OPTIONS to simply remove a file 
"asa_exit_anytime" which causes ASA to gracefully exit. In this project, ASA removes "asa_exit_anytime" each 47 hours. Accounts on supercomputers require such constraints.

\section{Path-Integral Methodology}

\subsection{Generic Applications}

There are many systems well defined by (a) Fokker-Planck/Chapman-Kolmogorov partialdifferential equations, (b) Langevin coupled stochastic-differential equations, and (c) Lagrangian or Hamiltonian path-integrals. All three systems of equations are mathematically equivalent, when limits of discretized variables are taken in the induced Riemannian geometry of the system due to nonlinear and time-dependent diffusions [31-34].

\subsubsection{Path-Integral Algorithm}

A classical system with $\mathrm{N}$ variables defined by a path integral is indexed by $i$, at multiple times indexed by $\rho$, defined by its Lagrangian $L$ :

$$
\begin{gathered}
P\left[q_{t} \mid q_{t_{0}}\right] d q(t)=\int \ldots \int D q \exp \left(-\min \int_{t_{0}}^{t} d t^{\prime} L\right) \delta\left(q\left(t_{0}\right)=q_{0}\right) \delta\left(q(t)=q_{t}\right) \\
D q=\lim _{u \rightarrow \infty} \prod_{\rho=1}^{u+1} g^{1 / 2} \prod_{i}(2 \pi \Delta t)^{-1 / 2} d q_{\rho}^{i} \\
L\left(\dot{q}^{i}, q^{i}, t\right)=\frac{1}{2}\left(\dot{q}^{i}-g^{i}\right) g_{i i^{\prime}}\left(\dot{q}^{i^{\prime}}-g^{i^{\prime}}\right)+R / 6 \\
g_{i i^{\prime}}=\left(g^{i i^{\prime}}\right)^{-1} \\
g=\operatorname{det}\left(g_{i i^{\prime}}\right)
\end{gathered}
$$

The diagonal diffusion terms are $g^{i i}$ and the drift terms are $g^{i}$. If the diffusions are not constant, there are additional terms in the drift, and in a Riemannian-curvature potential $R / 6$ for dimension $>1$ in the midpoint Stratonovich/Feynman discretization [33].

The path-integral approach is very useful to intuitively define physical variables from the Lagrangian $L$ in terms of its underlying variables $q^{i}$ :

$$
\begin{gathered}
\text { Momentum: } \Pi^{i}=\frac{\partial L}{\partial\left(\partial q^{i} / \partial t\right)} \\
\text { Mass: } g_{i i^{\prime}}=\frac{\partial L}{\partial\left(\partial q^{i} / \partial t\right) \partial\left(\partial q^{i^{\prime}} / \partial t\right)} \\
\text { Force: } \frac{\partial L}{\partial q^{i}} \\
\mathrm{~F}=\text { ma: } \delta L=0=\frac{\partial L}{\partial q^{i}}-\frac{\partial}{\partial t} \frac{\partial L}{\partial\left(\partial q^{i} / \partial t\right)}
\end{gathered}
$$

Canonical Momenta Indicators $\left(\mathrm{CMI}=\Pi^{i}\right)$ were used successfully in neuroscience [3537], combat analyses [38], and financial markets [35,39].

The histogram procedure numerically approximates sums of rectangles of height $P_{i}$ and width $\Delta q^{i}$ at points $q^{i}$. Considering a one-dimensional system in the prepoint Ito 
discretization, the path-integral representation is written in terms of the kernel $G$, for each intermediate integrals, as

$$
\begin{gathered}
P(x ; t+\Delta t)=\int d x^{\prime}\left[g^{1 / 2}(2 \pi \Delta t)^{-1 / 2} \exp (-L \Delta t)\right] P\left(x^{\prime} ; t\right) \\
=\int d x^{\prime} G\left(x, x^{\prime} ; \Delta t\right) P\left(x^{\prime} ; t\right) \\
\quad P(x ; t)=\sum_{i=1}^{N} \pi\left(x-x^{i}\right) P_{i}(t) \\
\pi\left(x-x^{i}\right)= \begin{cases}1, & \left(x^{i}-\frac{1}{2} \Delta x^{i-1}\right) \leq x \leq\left(x^{i}+\frac{1}{2} \Delta x^{i}\right) \\
0, & \text { otherwise }\end{cases}
\end{gathered}
$$

This yields

$$
T_{i j}(\Delta t)=\frac{2}{\Delta x^{i-1}+\Delta x^{i}} \int_{x^{i}-\Delta x^{i-1 / 2}}^{\substack{\left.P^{i}+\Delta t\right)=T_{i j}(\Delta t) P_{j}(t) \\ x^{i}+\Delta x^{i} / 2}} d x \int_{x^{j}-\Delta x^{j-1 / 2}}^{x^{j}+\Delta x^{j} / 2} d x^{\prime} G\left(x, x^{\prime} ; \Delta t\right)
$$

$T_{i j}$ is a banded matrix from the Gaussian short-time probability centered about the (possibly time-dependent) drift.

Explicit dependence of $L$ on time $t$ is included. Care developing the mesh $\Delta q^{i}$ yields diagonal elements of the diffusion matrix as

$$
\Delta q^{i} \approx\left(\Delta t g^{i i}\right)^{1 / 2}
$$

This constrains the covariance of each variable to be a (nonlinear) function of all variables, presenting a rectangular underlying mesh. Since integration is inherently a smoothing process [40], fitting data over the short-time probability distributions permits the coarser meshes than the corresponding stochastic differential equation(s). The coarser resolution is appropriate for numerical solutions of the time-dependent path integral. Consideration of contributions to the first and second moments gives conditions on the time and variable meshes [41]. A scan of the time slice can be determined by a scan of $\Delta t \leq \bar{L}^{-1}$, where $\bar{L}$ is the uniform/static Lagrangian, giving ranges of most important contributions to the probability distribution $P$.

\subsubsection{Direct Kernel Evaluation}

Several projects have used this algorithm [11,12,23,24,41,42]. 2-dimensional codes were developed for projects in Statistical Mechanics of Combat (SMC) [21], SMNI [23], and Statistical Mechanics of Financial Markets (SMFM) [22].

The 1-D PATHINT code was generalized by the author to $\mathrm{N}$ dimensions. A quantum generalization also was made to the code, changing all variables and functions to complex variables, changing about 7500 lines of PATHINT code. The $\mathrm{N}$-dimensional code was developed for classical and quantum systems [16-18]. 


\subsubsection{Monte Carlo vs Kernels}

Path-integral numerical applications often use Monte Carlo algorithms [43]. This approach includes the author's ASA code with ASA_SAMPLE OPTIONS [28]. This project, however, concerns time-sequential serial random shocks, which are not conveniently treated with Monte-Carlo/importance-sampling algorithms.

\subsection{Quantum Path Integral Algorithms}

\subsubsection{Scaling Issues}

qPATHINT has been tested with shocks to quantum options wave-functions [18], illustrating computational scaling issues described in the Performance and Scaling Section.

\subsubsection{Imaginary Time}

Imaginary-time Wick rotations transform imaginary-time real-variable time. When used with numerical calculations, multiple foldings of the path integral leaves no audit trail back to imaginary time (private communication with several authors of path-integral papers, including Larry Schulman on 18 Nov 2015) [34].

\section{SMFM With qPATHINT}

Above considerations define fitting a volatility of volatility options model with Classical algorithms, with qPATHINT numerically calculating the Quantum path-integrals at each time between epochs. At the beginning of each epoch, time is reset $(t=0)$ since the wavefunction is decohered ("collapsed") by a prior American stop-measurement; until the end of that epoch there are multiple calls to Classical functions calculating the evolution of the conditional short-time probability distribution.

\subsection{SMFM 2-D}

The two-factor model includes stochastic volatility $\sigma$ of the underlying $S$,

$$
\begin{gathered}
d S=\mu d t+\sigma F\left(S, S_{0}, S_{\infty}, x, y\right) d z_{S} \\
d \sigma=v d t+\epsilon d z_{\sigma} \\
<d z_{i}>=0, i=\{S, \sigma\} \\
<d z_{i}(t) d z_{j}\left(t^{\prime}\right)>= \begin{cases}d t \delta\left(t-t^{\prime}\right) \quad i=j \\
\rho d t \delta\left(t-t^{\prime}\right) \quad i \neq j\end{cases} \\
F\left(S, S_{0}, S_{\infty}, x, y\right)= \begin{cases}S, & S<S_{0} \\
S^{x} S_{0}^{1-x}, & S_{0} \leq S \leq S_{\infty} \\
S^{y} S_{0}^{1-x} S_{\infty}^{x-y}, & S>S_{\infty}\end{cases}
\end{gathered}
$$

where $S_{0}$ and $S_{\infty}$ lie outside the region that fits other parameters, e.g., $S_{0}=1$ and $S_{\infty}=$ 20 for Eurodollar futures which historically have very tight ranges relative to other markets. The Black-Scholes form $F=S$ inside $S<S_{0}$ is used so that no negative prices arise since the distribution is excluded from $S<0$. Put-call parity for European options is derived independent of mathematical modelling of options [44]. This is given by 


$$
c+X e^{-r(T-t)}=p+S
$$

where $c(p)$ is the fair price of a call (put), $X$ is the strike price, $r$ is the risk-free interest rate, $t$ is the present time, $T$ is the time of expiration, and $S$ is the underlying market. $y=0$ reflects a normal distribution defining ignorance of markets outside the range of $S>S_{\infty}$. This one-factor model assumes constant $\sigma$. Note that BS models incorrectly include contributions from large $S$ regions because of their fat tails [45].

The formula for pricing an option $P$, derived in a Black-Scholes generalized framework, leaving out interest-rate discounting, uses the form

$$
\begin{gathered}
d S=\mu S d t+\sigma F\left(S, S_{0}, S_{\infty}, x, y\right) d z_{S} \\
d \sigma=v d t+\epsilon d z_{\sigma}
\end{gathered}
$$

Some experimentation was performed with alternative functional forms, e.g., appling smooth cutoffs across the above three regions of $S . F^{\prime}$, a function $F$ designed to revert to the lognormal Black-Scholes model in several limits, was used

$$
\begin{gathered}
F^{\prime}\left(S, S_{0}, S_{\infty}, x\right)=S C_{0}+\left(1-C_{0}\right)\left(S^{x} S_{0}^{1-x} C_{\infty}+S_{0}\left(1-C_{\infty}\right)\right) \\
C_{0}=\exp \left[-\left(\frac{S}{S_{0}} \frac{|1-x|}{1+|1-x|}\right)^{|2-x|+1}\right] \\
C_{\infty}=\exp \left[-\left(\frac{S}{S_{\infty}}\right)^{2}\right] \\
\lim _{S \rightarrow \infty, x \neq 1} F^{\prime}\left(S, S_{0}, S_{\infty}, x\right)=S_{0}=\mathrm{constant} \\
\lim _{S \rightarrow 0^{+}} F^{\prime}\left(S, S_{0}, S_{\infty}, x\right)=\lim _{x \rightarrow 1} F^{\prime}\left(S, S_{0}, S_{\infty}, x\right)=S
\end{gathered}
$$

Fits were most sensitive to the data when the central region was simply $S^{x}$ using $F$ above.

\subsection{Two-Factor Volatility and PATHINT Modifications}

In the two-factor model, the mesh of $S$ would depend on $\sigma$ and cause some problems in any PATHINT grid to be developed in $S$ - $\sigma$.

For some time considerations have been made to handle the generic problem for $n$-factor multivariate systems with truly multivariate diffusions within the framework of PATHINT. In one case, taking advantage of the Riemannian invariance of the probability distribution as discussed above, transforms diffusions to have only "diagonal" multiplicative dependence [46-49]. Unfortunately, this leads cumbersome numerical problems with the transformed boundary conditions [23]. Another method, is to develop a tiling of diagonal meshes for each factor $i$ that often are suitable for off-diagonal regions in an $n$-factor system, e.g.,

$$
\begin{aligned}
& \Delta M_{k}^{i}=2^{m_{k}^{i} \Delta M_{0}^{i}} \\
& \Delta M_{0}^{i} \approx \sqrt{g_{k_{0}}^{|i||i|} \Delta t}
\end{aligned}
$$

where the mesh of variable $i$ at a given point labeled by $k$ is an exponentiation of 2 , labeled by $m_{k}^{i}$. The integral power $m_{k}^{i}$ approximates a diagonal mesh in the one-factor PATHINT mesh conditions, giving a minimal mesh $\Delta M_{0}^{i}$ in regions of the Lagrangian with most important contributions to the distribution. 
After the at-the-money BPV are scaled to be equivalent, there is not a very large change in the one-factor ATM Greeks. Not changing the functional dependence of the Lagrangian on $S$ and $\sigma$ has determined the meshes using a diffusion for the $S$ equation as $\sigma_{0} F\left(S, S_{0}, S_{\infty}, x, y\right)$, where $\sigma_{0}$ is determined by the same BPV-equivalent condition as imposed on the one-factor models. This works well, taking the $\sigma$ equation with a limited range of influence in the calculations.

\subsection{Previous XSEDE SMFM Project}

It seems that "if" QM is not much of an issue. When QM does arrive, options on quantum markets will be required for hedging and speculation.

\subsubsection{Options Calculations}

A value of 9 off-diagonal terms are used on each side of the diagonal kernel. The model has noise of $S^{x}$, where $S$ is the underlying price and $x$ is an exponent. The underlying price is taken to be 7.0. A strike value of 7.5 is used for this table. The risk-free rate is taken to be 0.0675 . The cost of carry is taken to be 0 . A daily volatility of 0.00793725 is used, and this parameter is taken to be real for both PATHINT and qPATHINT.

There is no additional drift, but drift arises from the nonlinear noise [22,42]. In this context, shocks affect Greeks with "p" severely, where "p" denotes additional orders of derivatives. E.g., VegapPI (second derivative of $\Upsilon$ with respect to volatility) is very sensitive to shocks in this drift.

Results are given in a previous paper [18].

\subsubsection{Current Project}

ASA is used to fit volatility of volatility options over short-time conditional probability distributions, similar to previously projects [22], but now these forms are based on Quantum, instead of Classical, money.

\section{Performance and Scaling}

Code is used from a previous XSEDE grant "Quantum path-integral qPATHTREE and qPATHINT algorithm”, for qPATHINT runs.

\subsection{SMNI Scaling Estimates}

SMNI estimates were made on XSEDE.org's Expanse using 'gcc -O3', and for the onedimentional system.

SMNI uses a variable mesh covering 1121 points along the diagonal, with a maximum off-diagonal spread of 27; corners require extra CPU to care for boundaries. Also, oscillatory wave functions require large off-diagonal spreads [17].

$d t=0.0002$ requires 10 foldings of the distribution. This runs in $0.002 \mathrm{~s}$, giving $0.0002 \mathrm{~s} / \mathrm{qIteraction}$. With ' $\mathrm{g}$ ' the code takes $0.004 \mathrm{~s}$ to run. 


\subsection{Scaling Estimates N-D}

An $\mathrm{N}$-factor model with the same 1-D system cloned in all dimensions (each unit is a "double complex") was used for benchmarks:

$\mathrm{D}=1$ :imxall: 27 , jmxall: 7 , ijkcnt: 189

$\mathrm{D}=2$ :imxall: 729 , jmxall: 49 , ijkcnt: 35721

D=3:imxall: 19683 , jmxall: 343 , ijkcnt: 6751269

D=4:imxall: 531441 , jmxall: 2401 , ijkent: 1275989841

D=5:imxall: 14348907 , jmxall: 16807 , ijkcnt: 241162079949

D=6:imxall: 387420489 , jmxall: 117649 , ijkcnt: 45579633110361

D=7:imxall: 10460353203 , jmxall: 823543 , ijkcnt: 8614550657858229

The size of the kernel size $(\mathrm{I} \mathrm{J})^{\mathrm{N}}$, where $\mathrm{I}=$ imxall, $\mathrm{J}=$ jmxall $(=$ kernel band width), and kernel size $=\mathrm{ijkcnt}$.

The 2-D problem takes on the order of 200 times the 1-D problem. When the length of time is an issue, e.g., for dimensions $>2$, fits of drifts and covariance matrices to parameterized forms is a very good option [50].

\section{Conclusion}

Pilot studies give rationales for developing this particular kernel-based quantum path-integral algorithm. This can study serial random shocks that occur in many real systems. qPATHINT can be used for many quantum systems which are becoming increasingly important.

Acknowledgements: The author thanks the Extreme Science and Engineering Discovery Environment (XSEDE.org), for yearly supercomputer grants since February 2013.

\section{References}

1. L. Ingber, Revisiting our quantum world. In Hooke, A. (Ed.), Technological Breakthroughs and Future Business Opportunities in Education, Health, and Outer Space, IGI Global, Hershey, PA. 1-16 (2021)

2. M. Benedetti, E. Lloyd, S. Sack, M. Fiorentini, Parameterized quantum circuits as machine learning models. Quantum Science and Technology, 4(4) (2019)

3. S. Chakrabarti, R. Krishnakumar, G. Mazzola, N. Stamatopoulos, S. Woerner, W. Zeng, A threshold for quantum advantage in derivative pricing. Technical Report arXiv:2012.03819v2 [quant-ph], Goldman Sachs, New York, NY (2020)

4. S. Aaronson, P. Christiano, Quantum money from hidden subspaces. Technical Report arXiv:1203.4740 [quant-ph], MIT, Cambridge, MA (2012)

5. L. Accardi, A. Boukas, The quantum black-scholes equation. Technical Report arXiv:0706.1300 [q-fin.PR], U di Roma Torvergata, Rome (2007)

6. B. Baaquie, C. Coriano, M. Srikant, Quantum mechanics, path integrals and option pricing: Reducing the complexity of finance. Technical Report arXiv:condmat/0208191 [cond-mat.soft], National U Singapore, Singapore (2002)

7. K. Bartkiewicz, A. Cernoch, G. Chimczak, K. Lemr, A. Miranowicz, F. Nori, Experimental quantum forgery of quantum optical money. Technical Report arXiv:1604.04453v1 [quant-ph], Adam Mickiewicz University, Poznan, Poland (2016) 
8. J. Jogenfors, Quantum bitcoin: An anonymous and distributed currency secured by the no-cloning theorem of quantum mechanics. Technical Report arXiv:1604.01383 [quant-ph], Linkoping U, Linkoping, Sweden (2016)

9. K. Meyer, Extending and simulating the quantum binomial options pricing model. Technical Report Thesis, U Manitoba, Winnipeg, Canada (2009)

10. E. Piotrowski, M. Schroeder, A. Zambrzycka, Physica A 368(1), 176-182 (2005)

11. M. Wehner, W. Wolfer, Physical Review A 27, 2663-2670 (1983)

12. M. Wehner, W. Wolfer, Physical Review A 28, 3003-3011 (1983)

13. L. Ingber, Computational algorithms derived from multiple scales of neocortical processing. In Pereira, A. , Massad, E., Bobbitt, N. (Eds.), Pointing at Boundaries: Integrating Computation and Cognition on Biological Grounds, Springer, New York. 1-13 (2011)

14. L. Ingber, Columnar EEG magnetic influences on molecular development of shortterm memory. In Kalivas, G., Petralia, S. (Eds.), Short-Term Memory: New Research, Nova, Hauppauge, NY. 37-72 (2012)

15. L. Ingber, Sci 1(7), 1-21 (2018)

16. L. Ingber, International Journal of Innovative Research in Information Security 3(5), 1-15 (2016)

17. L. Ingber, International Journal of Innovative Research in Information Security 4(2), 14-22 (2017)

18. L. Ingber, International Journal of Innovative Research in Information Security 4(2), 7-13 (2017)

19. L. Ingber, The Open Cybernetics Systemics Journal 11, 119-133 (2017)

20. L. Ingber, Forecasting COVID-19 with importance-sampling and path-integrals. WSEAS Transactions on Biology and Biomedicine (to be published)

21. L. Ingber, H. Fujio, M. Wehner, Mathematical Computer Modelling 15(1), 65-90 (1991)

22. L. Ingber, Physica A 283(3-4), 529-558 (2000)

23. L. Ingber, P. Nunez, Physical Review E 51(5), 5074-5083 (1995)

24. L. Ingber, R. Srinivasan, P. Nunez, Mathematical Computer Modelling 23(3), 43-53 (1996)

25. L. Ingber, J. Wilson, Mathematical Computer Modelling 31(8/9), 167-192 (2000)

26. L. Ingber, C. Chen, R. Mondescu, D. Muzzall, M. Renedo, Probability tree algorithm for general diffusion processes. Physical Review E 64(5), 056702-056707 (2001)

27. L. Ingber, Mathematical Computer Modelling 12(8), 967-973 (1989)

28. L. Ingber, Adaptive simulated annealing (ASA). Technical Report Global optimization C-code, Caltech Alumni Association, Pasadena, CA (1993)

29. L. Ingber, Adaptive simulated annealing. In Oliveira, H., Petraglia, A., Ingber, L., Machado, M., Petraglia, M. (Eds.), Stochastic global optimization and its applications with fuzzy adaptive simulated annealing, Springer, New York. 33-61 (2012)

30. L. Ingber, Hybrid classical-quantum computing: Applications to statistical mechanics of neocortical interactions. Technical Report Report 2021:HCQFA, Physical Studies Institute, Ashland, OR (2021)

31. L. Ingber, Physica D 5, 83-107 (1982)

32. L. Ingber, Physical Review A 28, 395-416 (1983) 
33. F. Langouche, D. Roekaerts, E. Tirapegui, Functional Integration and Semiclassical Expansions. Reidel, Dordrecht, The Netherlands (1982)

34. L. Schulman, Techniques and Applications of Path Integration. J. Wiley \& Sons, New York (1981)

35. L. Ingber, In Amari, S.I. , Xu, L., King, I., Leung, K.S. (Eds.), Progress in Neural Information Processing, Springer, New York. 777-784 (1996)

36. L. Ingber, Physical Review E 55(4), 4578-4593 (1997)

37. L. Ingber, Mathematical Computer Modelling 27(3), 33-64 (1998)

38. M. Bowman, L. Ingber, Canonical momenta of nonlinear combat. In Proceedings of the 1997 Simulation Multi-Conference, 6-10 April 1997, Atlanta, GA, Society for Computer Simulation, San Diego, CA, 1-6 (1997)

39. L. Ingber, R. Mondescu, IEEE Transactions Neural Networks 12(4), 776-790 (2001)

40. L. Ingber, Physical Review A 42(12), 7057-7064 (1990)

41. M. Wehner, W. Wolfer, W. Physical Review A 35, 1795-1801 (1987)

42. L. Ingber, J. Wilson, Mathematical Computer Modelling 29(5), 39-57 (1999)

43. M. O’Callaghan, B. Miller, Physical Review E 89(042124), 1-19 (2014)

44. J. Hull, Options, Futures, and Other Derivatives, Third Edition. Prentice Hall, Upper Saddle River, NJ (1997)

45. F. Fabozzi, Treasury Securities and Derivatives. Fabozzi Associates, New Hope, PA (1998)

46. L. Ingber, Physical Review E 49(5B), 4652-4664 (1994)

47. F. Fabozzi, Treasury Securities and Derivatives. Fabozzi Associates, New Hope, PA (1998)

48. J. Preskill, Quantum mechanics. Technical Report Lecture Notes, Caltech, Pasadena, CA (2015)

49. S. Wiesner, SIGACT News 15(1), 78-88 (1983)

50. L. Ingber, Virtual Economics 3(2), 7-24 (2020) 